This as far as possible, is the exact description of the scalpel. It has a long handle so that it will be well supported during use. The blade is put at an angle of $120^{\circ}$ to the handle, obviously to combat all the difficulties mentioned above. You can now work freely with it. Neither the nose of the patient, nor spatula, nor the assistant's hand will be in the way because, owing to this angle, your working hand will be on a far higher level than all of them, and there is no need for it to lie on the flat. It has both sides sharp so as to work well for the right as for the left lids, for the upper as for the lower lids, and with your right as with your left hand, though with this scalpel, there is no need for you to use your left hand at all. With this bent blade, whose sides and tip are sharp, you can reach the most medial parts of both lids as easily and precisely as any other part of the lid, without any discomfort, risk, or loss of time. Thus it facilitates the operation immensely, adding so much to the precision in its doing, and consequently in its success surgically and cosmetically.

Besides its use in this operation, the new scalpel can be used in the opening of styes and chalazia, as well as many other minor operations. In exision of lashes it is really far better and easier to use than any other scalpel.

The design and description of the knife, and the points in its favour, were sent by me to the firm of John Weiss \& Son, Ltd., 287, Oxford Street, London, who after appreciating the idea and finding that there is no practical difficulty in the design, were kind enough to manufacture the knife.

I am putting the knife for trial and I would really be much obliged to any of our colleagues who will be kind enough to report me his experience with it after its use in his cases, and hope that it will fulfil all it is meant for with you as it did with me.

\title{
ANNOTATION
}

\section{The Tuberculous Phlycten}

Dr. Dorothy Price, in the Irish Journal of Medical Science, July, 1940 , contributes a short note on the tuberculous phlycten from the point of view, not of the ophthalmologist, but of the clinician.

At the present time opinion, based on published statistics, is agreed that from 85 to 95 per cent. of phlyctenular cases show a positive "tuberculous reaction," and are considered to be due to a tuberculin allergy. The clinician finds that the tuberculous phlycten is an allergic phenomenon; it arises first during the primary and hypersensitive stage of pulmonary tuberculosis in a very small proportion 
of the cases so infected. Erythema nodosum is another such tuberculous allergic phenomenon: it appears at the beginning of the first stage and coincides with the initial fever. The phlycten appears at an interval of time after the initial fever, probably two months or more, and is, therefore, a rather late manifestation of primary lung infection.

This comparatively late appearance may account for the fact that many of these cases on X-ray examination fail to show signs of pulmonary infection; fresh phlyctens are usually found to be associated with residual root gland enlargement, without evidence of focus in the lung field.

In Dublin phlyctens occur most commonly during the 1-4 age period; they may of course appear later. Erythema nodosum, on the other hand, is more commonly seen in adolescents and is extremely rare in the first years of life.

With regard to general treatment, Dr. Price insists that all cases should have immediate investigation of the underlying tuberculous condition before any but local treatment is given for the eyes. Institutional treatment without delay in open-air preventorium or children's sanatorium is ideal for early cases; failing these, rest for a certain period in the childrens' ward of a hospital is next best. Rest is even more important than fresh air and liberal diet, for it is the author's experience that the two latter, when associated with over-fatigue, have never effected a rapid cure. Cases of longer standing, showing residual enlargement of the hilar glands, may be allowed rather more relaxation than the "half-time rest" recommended for cases of early infection. The child, in this, rises at 11 a.m. and goes to bed at 5 p.m., the intervening hours are spent as far as possible in the fresh air, exercise being restricted. As regards special treatment Dr. Price says that ultra-violet rays are contraindicated in all cases of primary lung tuberculosis in children. Operative interference, such as tonsillectomy is also contra-indicated. She has seen (clinically and radiologically) re-activation of hilar glands after both these therapeutic measures in cases where the primary tuberculous glands had just begun to heal up nicely. Tuberculin therapy should never be given during primary tuberculosis of the lungs in children (nor in adults). It may be employed with advantage for the eye condition when primary focus and glands are healed; four years should be a safe interval after healing. "A phlycten, which resists treatment or recurs when a long interval has passed since the child healed his primary lung infection, will yield to tuberculin, given by intra-dermal injection in very small doses, and continued over a long period."

As ophthalmologists we are often so immersed in the local condition that the general state tends to be overlooked or underestimated. The other side of the picture as given here should be of great value to all of us. 